\title{
Multidisciplinary Considerations in the Design of Wings and Wing Tip Devices
}

Andrew Ning

Brigham Young University - Provo, aning@byu.edu

Ilan Kroo

Stanford University

Follow this and additional works at: https://scholarsarchive.byu.edu/facpub

Part of the Mechanical Engineering Commons

\section{Original Publication Citation}

Ning, A., and Kroo, I., "Multidisciplinary Considerations in the Design of Wings and Wing Tip Devices," Journal of Aircraft, Vol. 47, No. 2, Mar. 2010, pp. 534-543. DOI: 10.2514/1.41833

\section{BYU ScholarsArchive Citation}

Ning, Andrew and Kroo, Ilan, "Multidisciplinary Considerations in the Design of Wings and Wing Tip Devices" (2010). Faculty Publications. 1629.

https://scholarsarchive.byu.edu/facpub/1629

This Peer-Reviewed Article is brought to you for free and open access by BYU ScholarsArchive. It has been accepted for inclusion in Faculty Publications by an authorized administrator of BYU ScholarsArchive. For more information, please contact ellen_amatangelo@byu.edu. 


\title{
Multidisciplinary Considerations in the Design of Wings and Wing Tip Devices
}

\author{
S. Andrew Ning* and Ilan Kroo ${ }^{\dagger}$ \\ Stanford University, Stanford, CA, 94305
}

\begin{abstract}
Conceptual wing design analysis methods are combined with numerical optimization to find minimum drag wings subject to constraints on lift, weight, pitching moment, and stall speed. Tip extensions and winglets designed for minimum drag achieve similar performance, with the optimal solution depending on the ratio of the maneuver lift coefficient to the cruise lift coefficient. The results highlight the importance of accounting for the depth of the wing structural box in the weight model, and including constraints on stall speed. For tailless aircraft, C-wings show a slight performance advantage over wings with winglets when longitudinal trim and stability constraints are considered. This performance advantage is more significant for span-constrained or low sweep designs. Finally, to demonstrate other possible applications of the method, planar wings with active load alleviation are optimized, showing drag savings on the order of $15 \%$.
\end{abstract}

\section{Nomenclature}

$\begin{array}{ll}A R & \text { aspect ratio } \\ b & \text { span } \\ C_{L} & \text { lift coefficient } \\ c_{l} & \text { section lift coefficient } \\ C_{m a c} & \text { pitching moment coefficient about the aerodynamic center } \\ C_{m} g & \text { pitching moment coefficient about the center of gravity } \\ c_{r} & \text { root chord } \\ c_{t} & \text { tip chord } \\ D & \text { drag } \\ e & \text { span efficiency } \\ I_{b} & \text { bending weight index } \\ J & \text { objective } \\ L & \text { lift } \\ \ell & \text { length } \\ M_{b} & \text { bending moment } \\ S & \text { wing area } \\ \text { s.m. } & \text { static margin } \\ t & \text { section thickness } \\ t_{s} & \text { skin thickness } \\ V_{s} & \text { stall speed } \\ W & \text { weight } \\ \text { Subscripts } & \\ \text { max } & \text { maximum } \\ \text { mvr } & \text { maneuver } \\ \text { ref } & \text { reference } \\ \end{array}$

*Ph.D. Candidate, Department of Aeronautics and Astronautics, AIAA Student Member.

${ }^{\dagger}$ Professor, Department of Aeronautics and Astronautics, AIAA Fellow. 


\section{Introduction}

High fuel costs and environmental concerns provide continuing motivation for research aimed at increasing aircraft efficiency. Vortex drag is a major contributor to aircraft drag, typically accounting for about $40 \%$ of the drag in cruise and about $80-90 \%$ of the drag in second segment climb. ${ }^{1}$ Since wing tip geometry strongly affects vortex drag, wing tip optimization has received a great deal of attention. Improved wing tip design can not only benefit new designs, but can also improve performance of existing wings through retrofits.

One of the early wing tip modifications was a simple end plate. ${ }^{2,3}$ These end plates were shown to increase the effective span of the wing, thus reducing the vortex drag. Richard Whitcomb at NASA achieved greater gains in efficiency through carefully designed high aspect ratio end plates which he termed winglets. His experimental data published in 1976 showed that a winglet improved the lift to drag ratio by nearly a factor of two as compared to a tip extension. ${ }^{4}$

Many studies since then, both computation and experimental, compare tip extensions with winglets. ${ }^{5-11}$ The conclusions differ depending on the structural constraint, whether or not viscous drag is included, and the range of geometries explored. There are several reasons why a reexamination of the relative benefits of tip extensions and winglets is warranted.

First, the structural constraint used in most of these studies is a fixed root bending moment. Root bending moment does not account for the effect of chordwise changes in the planform on the bending weight. A fixed root bending moment can be especially inappropriate for nonplanar configurations, as large winglets can be designed with zero or even negative changes in root bending moment. Also, most previous studies apply the weight constraint at the cruise lift coefficient (or at some other fixed lift coefficient). However, the results are sometimes significantly different as the critical structural lift coefficient is varied for a given wing design (corresponding to different critical structural altitudes).

Second, many of these studies compare only induced drag. Including viscous drag can be particularly important when comparing tip extensions with winglets, as the designs often have very different wetted areas. This is often addressed by keeping the wetted area of the entire wing constant. However, for nonplanar wings, neither fixing the wetted area nor fixing the projected area allows for fair comparisons between designs because of tradeoffs with viscous drag and maximum lift capabilities.

Third, all of these studies analyze a small number of designs, or parametrically vary a few of the design variables. Wings with tip devices introduce more planform variables than can be handled properly by simple trade studies. In particular, many of the above mentioned studies use simple linearly extrapolated tip extensions. Such a tip extension is an ineffective use of wetted area for the same reason why an end plate is less effective than a winglet. Optimization is employed in some of the previous studies, but is limited to optimizing the lift distribution only.

This paper seeks to address these concerns, while still retaining simplicity in the analysis methods, as appropriate for conceptual design studies. Certainly, the choice of a tip extension, winglet, or other tip device depends upon many other considerations not addressed here such as the effect on the aircraft stability and control, aeroelastics, and even marketing. Our objective in this paper is not to design an airplane, rather we seek to explore fundamental, but complex multidisciplinary considerations in the design of wings and wing tip devices. We will not conclude whether winglets or tip extensions are "better", as this would require a specific and complete aircraft design study. Instead, the results show the importance of various assumptions and design degrees of freedom.

First, tip extensions and winglets are compared using nonlinear optimization. C-wings are then investigated to determine if there are significant performance advantages to $\mathrm{C}$-wing designs as compared to wings with winglets. Finally, as an example of the wider applicability of the method, a planar wing with active 
load alleviation is optimized.

\section{Method}

\section{A. Fundamental Considerations}

Wing design involves fundamental tradeoffs between drag, weight, and stall speed. Fixing the area of the wing is often done to simplify some of the interactions in simple trade studies, but even then the tradeoffs are not always clear. As a simple example, consider adding a winglet to a wing and increasing its height. If the wetted area of the wing/winglet combination is fixed, then viscous drag and area-dependent weight can be kept roughly constant while induced drag is reduced. However, the bending weight increases not only due to the increased height of the winglet, but also due to the smaller chords in the main wing. In addition, the projected area decreases resulting in decreased maximum lift capabilities and higher stalls speeds. Alternatively, the projected area of the wing may be fixed. This will allow for the stall speed performance to be roughly maintained while induced drag is decreased, but the viscous drag will increase as will both the area-dependent and load-dependent weight of the wing.

These fundamental tradeoffs can have complex interactions, especially as the wing design problem includes additional degrees of freedom. Nonlinear optimization allows the relative benefits of wing designs to be assessed more completely. In this paper we consider wings designed for minimum drag with fixed lift, weight, and stall speed. The methods used for computing drag, weight, and stall speed are outlined below. Validation of the methodology is done by comparing to a variety of analytic solutions, ${ }^{12}$ as well as to past numerical studies. ${ }^{8,13}$ Parameters such as panel density are chosen to ensure that results are within a percent or two of these known solutions. These results, along with further implementation details, can be found in our previous paper. ${ }^{14}$

\section{B. Drag Model}

This study investigates only low speed wings. For these cases, only vortex drag and viscous drag need to be considered. More realistic configurations would also need to include compressibility drag. Vortex drag is calculated at the Trefftz plane by using a drag free wake leaving the trailing edge of the wing planform. Viscous drag is calculated using strip theory by assuming a parabolic variation in viscous drag coefficient with section lift coefficient. Changes in loading with twist and angle of attack are computed using a discrete vortex Weissinger model. ${ }^{15}$ Fifty panels are equally spaced across the wing semi-span. The methodology is similar to that used by Kroo. ${ }^{8}$

\section{Weight Model}

The wing weight is decomposed into a part due to the load, and a part due to the size of the wing. Areadependent variations in wing weight are particularly important to consider for nonplanar configurations.

$$
W=W_{\text {load }}+W_{\text {area }}
$$

The load dependent weight is assumed to be proportional to the volume of material required to support a fully stressed wing in bending (a good approximation for wings of moderate to high aspect ratios where differences in shear-dependent weight are negligible, and for wings with low to moderate sweep where differences in torsion-dependent weight are negligible), and that the area-dependent weight is proportional to the gross area of the wing. The load dependent weight is given by

$$
I_{b}=\int \frac{\left|M_{b}\right|}{t} d s
$$

which is integrated along the main structural spar. Wing weight is then given by

$$
W=k_{1} I_{b}+k_{2} S
$$

where $k_{1}$ and $k_{2}$ are appropriately chosen constants. In this paper we are only interested in computing the ratio of weight to the reference weight, which leaves only one undetermined coefficient.

$$
\frac{W}{W_{\text {ref }}}=\frac{k_{1} I_{b}+k_{2} S}{k_{1} I_{\text {bref }}+k_{2} S_{\text {ref }}}=\frac{I_{b}+k_{W} S}{I_{\text {ref }}+k_{W} S_{\text {ref }}}
$$


where $k_{W}=k_{2} / k_{1}$. The value of this constant was determined by correlating aircraft data with the bending index used here $\left(k_{W}=3.57 \times 10^{4}\right)$.

It should be mentioned that the value for wing weight does not represent an actual dimensional wing weight, but is intended to scale appropriately with changes in wing planform and aerodynamic loading in order to compare the wing weight of different designs. A wing weight estimate of this form has been shown to correlate well with the wing weight data from actual transport aircraft. ${ }^{a}$

One additional modification is made to the weight computation to account for the difference in cruise loading and critical structural loading. Here, we assume that the aircraft is maneuver critical rather than gust critical. In this case the maximum load factor may be the same for many different aircraft, but the critical structural altitude will vary. This means that the maneuver lift coefficient (at which the bending weight is computed) must be allowed to be different from the cruise lift coefficient. For a given geometry, the load dependent weight index can be shown to be a function only of the ratio of the maneuver lift coefficient to the cruise lift coefficient. ${ }^{14}$

The ratio $C_{L m v r} / C_{L}$ relates the critical structural altitude to the cruise altitude. For wider applicability of the results, cases are shown for $C_{L m v r} / C_{L}$ equal to 1 and 2.5. As a specific example, and that used in this paper, assume a fixed load factor of 2.5 (many transport aircraft have their maximum maneuver load factor set at 2.5 as per FAR Part $25^{16}$ ). Then, if the cruise altitude of the aircraft is $35,000 \mathrm{ft}, C_{L m v r} / C_{L}=2.5$ corresponds to a critical structural altitude of $35,000 \mathrm{ft}$, whereas $C_{L m v r} / C_{L}=1$ corresponds to a critical structural altitude of $14,000 \mathrm{ft}$ at fixed Mach number. Thus, the choice of these two values is meant to capture differences in designs over a wide range of critical structural altitudes.

While wing bending loads are clearly important, a more complete configuration study would also need to consider other structural loading conditions such as those provided by inertial relief, taxi-bump, etc. Related constraints such as fuel volume requirements, and ground strike deflection would also be important in the structural design. Most of these considerations require detailed information about mass properties, fuel distributions, and performance requirements appropriate for a specific aircraft design study. However, the purpose of this paper is to emphasize the fundamental tradeoffs between load distribution, weight, and drag. The metric used here for wing weight is only intended to capture the dominant effects of wing planform and aerodynamic loading changes on wing weight.

\section{Stall Speed Model}

Stall speed is given by

$$
V_{s}=\sqrt{\frac{2 L}{\rho S C_{L \max }}}
$$

A constraint on stall speed is important when optimizing the planform geometry. Without this constraint, optimization often leads to unrealistically small tip chords with high section lift coefficients. All designs are required to have a stall speed less than or equal to that of the reference wing. Since the lift is fixed, this gives the inequality constraint $C_{L \max } S \geq\left.\left(C_{L_{\max }} S\right)\right|_{\text {ref }}$.

$C_{L \max }$ is difficult to predict and is really a three dimensional, unsteady, viscous phenomena. Consistent with the simplicity of the aero/structural model, $C_{L \max }$ is estimated simply from critical section theory. Essentially, this means that stall is predicted when any one section of the wing reaches a specified section $c_{l \max }$. Since no high-lift devices are modeled, the clean wing stall speed is constrained.

This constraint on stall speed is not directly imposed in the optimization problem. For a given wing area the required $C_{L \max }$ can be found directly from the constraint $C_{L \max } S \geq\left.\left(C_{L \max } S\right)\right|_{\text {ref }}$. The constraint imposed in the optimization problem is that at the required $C_{L \max }$ the $c_{l}$ distribution must be everywhere less than or equal to a specified maximum section lift coefficient $\left(\left.\left\{c_{l}\right\}\right|_{C_{L \max }} \leq\left\{c_{l \max }\right\}\right.$.). In the results shown in this paper the vector $\left\{c_{l \max }\right\}$ is constant across the wing for simplicity in discussing the results.

\section{E. Optimization}

There are three basic approaches to this optimization problem, each of increasing complexity over the previous. The first is the method of restricted variations. This method allows for analytic solutions of optimal normalwash distributions by considering small perturbations to the lift distribution. Consideration for parasitic drag, section lift coefficient constraints, and weight constraints can even be included. The

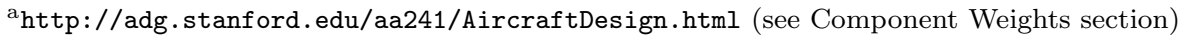


analytic solutions allow for good insight, but the method is restricted to simple geometries with simple formulations for the objective and constraints.

The second option also optimizes the lift distribution, but can allow for more complex geometries and constraints. Under the assumptions of the Prandtl-Glauert equation, the optimization can be posed as a quadratic programming problem. If only equality constraints are included, like lift and weight, then optimal loading is given directly by solving a linear system of equations. This approach has been used many times in the past. ${ }^{8,11,17}$ This method is good for giving insight into the effect of different weight models, comparing results with and without viscous drag, and for performing trade studies with planform variations. The rapid nature of the load optimization problem allows for trades studies with a fairly large number of wing designs.

The method may also be extended by including linear inequality constraints like stall speed. In these cases the optimization problem is still a convex quadratic programming problem which is easily solved. This approach has been pursued in some of our past studies. ${ }^{14}$

The third option, and the one used in this paper, is to also include all the planform parameters as design variables. In this case, the objective and constraints are nonlinear functions of the design variables. However, the functions are still smooth allowing for gradient based methods. Furthermore, the sensitivities of the objective and constraints to the additional design variables are approximated quite well by piecewise linear functions over a broad range of step sizes, allowing for robust and quick convergence using finite differences. The results shown in this paper are all solved using the sequential quadratic programming method.

\section{Nonlinear Planform Optimization}

Four different optimization cases are presented: tip geometry for a retrofitted wing, tip geometry for an optimized wing, C-wings with stability and trim constraints, and planar wings with active load alleviation. We emphasize again that the results are intended to explore the effect of multidisciplinary considerations in wing design, they are not intended to represent complete configuration design studies.

\section{A. Tip Extensions and Winglets - Retrofit}

Whitcomb's study on winglets ${ }^{4}$ is a classic paper showing significant performance benefits by retrofitting a wing with a winglet as opposed to retrofitting with a tip extensions. It is of interest to re-compare the relative performance of tip extensions and winglets using optimized tip devices. The baseline wing used in this study is the same as that used by Whitcomb, ${ }^{4}$ representative of early transport wings (Figure 1). The planform parameters are summarized in Table 1. Figure 1 also shows the tip extension tested by Whitcomb, whereas Figure 2 shows the winglet tested by Whitcomb. These two figures are taken from Whitcomb's paper $^{4}$ with some modification for clarity.

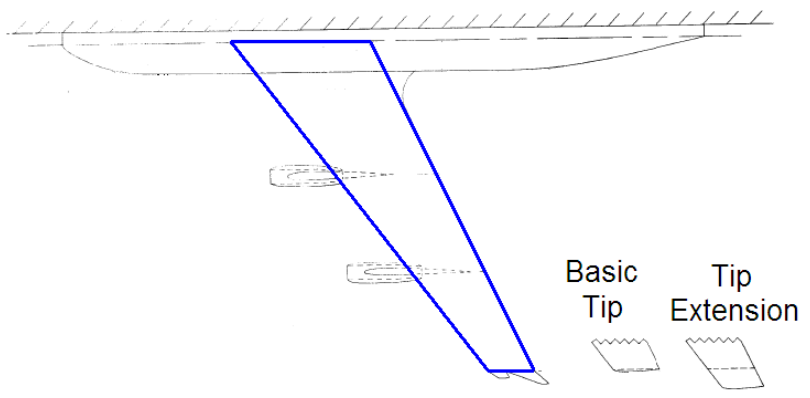

Figure 1. Geometry for baseline wing and tip extension used in Whitcomb study.

Table 1. Baseline wing

\begin{tabular}{|c|c||c|c|}
\hline $\mathrm{AR}$ & 7 & $\Lambda$ & $35^{\circ}$ \\
\hline $\mathrm{b}$ & $135 \mathrm{ft}$ & $\lambda$ & 0.35 \\
\hline
\end{tabular}




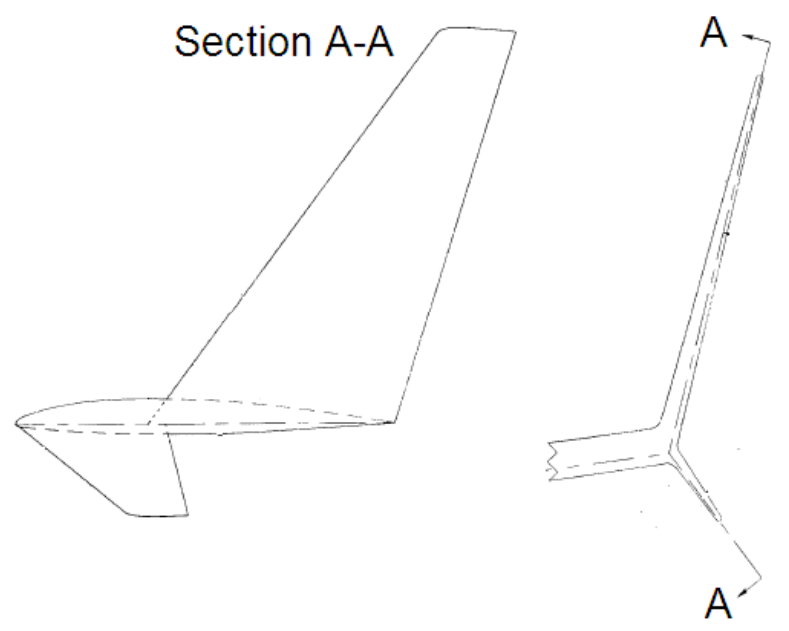

Figure 2. Geometry for winglet used in Whitcomb study.

The objective of this investigation is to find the minimum drag trapezoidal tip device for this fixed wing planform. Since only the tip geometry is optimized, the number of design variables is relatively small. These include the length, root chord, tip chord, and quarter chord sweep of the tip geometry. Twist is also optimized across the wing and tip. Twist is defined at three sections across the wing, and two across the tip with a linear distribution of twist in between. The twist definition locations across the wing are at the root, $30 \%$ semi-span, and tip, whereas the twist locations for the tip device are at its root and tip. The dihedral angle of the tip device is fixed at discrete values to facilitate comparison between tip extensions and winglets.

The optimization problem is subject to constraints on lift, weight, and stall speed. Rather than constrain the wing to a particular weight, the target weight is varied in order to create a Pareto front of optimal solutions trading off drag and weight.

The optimization problem is posed as follows:

$$
\begin{array}{cl}
\text { min. } & J=\frac{D}{D_{\text {ref }}} \\
\text { w.r.t. } & \text { wing: }\left\{\theta_{1,2,3}\right\}, \text { tip }:\left\{\ell, c_{r}, c_{t}, \Lambda, \theta_{4,5}\right\} \\
\text { s.t. } & 1-\frac{L}{L_{\text {ref }}} \leq 0 \\
\frac{W}{W_{\text {target }}}-1 \leq 0 \\
\left.\left\{c_{l}\right\}\right|_{C_{\text {Lmax }}}-\left\{c_{\text {lmax }}\right\} \leq 0 \\
0.01<\frac{\ell}{b / 2}<0.65 \\
0.01<\frac{c_{r}}{c_{\text {twing }}}<1 \\
0.01<\frac{c_{t}}{c_{t \text { ting }}}<1 \\
0^{\circ}<\frac{1}{<}<40^{\circ} \\
-20^{\circ}<\theta_{1-5}<20^{\circ}
\end{array}
$$

If sweep were allowed to vary freely, torsion-dependent weight and steady aeroelastics would need to be included. These have been explored in some computations, but for the present purposes they only complicate the problem formulation without providing much additional insight. Here, the tip sweep angle is not allowed to exceed $40^{\circ}$. The section maximum lift coefficient, $c_{l \max }$, is set equal to that of the reference wing.

There is one subtlety in the optimization procedure. The winglet length is a continuous design variable, but our analysis method uses a discrete vortex panel method. For numerical accuracy the panel size across 
the wing and tip is held constant. However, with a fixed panel size, the number of panels on the tip will vary discontinuously. This is a problem for gradient based optimization, but is resolved by varying the tip length parametrically.

Figures 3(a) and 3(b) show the Pareto fronts at a $C_{L m v r} / C_{L}$ of 1 and 2.5 respectively. Also shown on these figures are a few of the optimized geometries to give a sense of relative size. The values shown in the figures denote the length of the tip normalized by the wing semi-span (i.e. for a tip extension, a value of 0.14 corresponds to a $14 \%$ span increase).

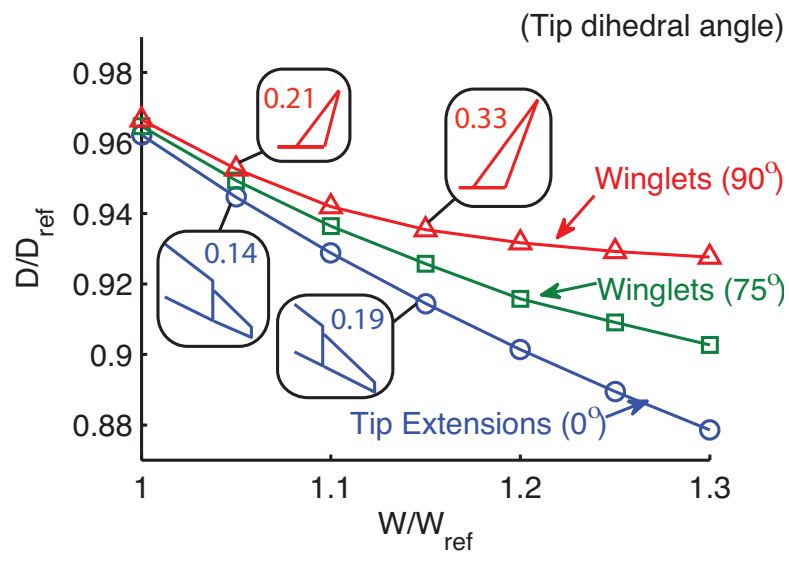

(a) $C_{L m v r} / C_{L}=1.0$, tip extensions optimal

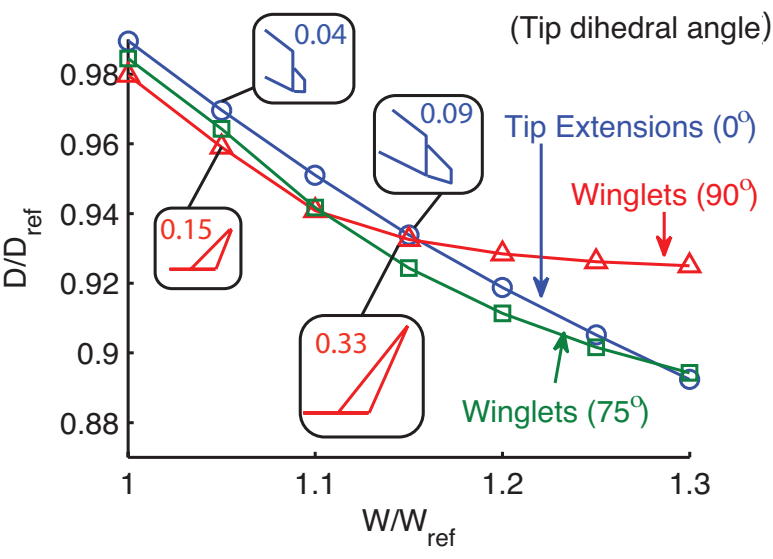

(b) $C_{L m v r} / C_{L}=2.5$, winglets optimal

Figure 3. Pareto front of optimal tip geometries with relative sizes also shown.

The figures show that for minimum drag the choice of tip extension versus winglet depends upon the ratio $C_{L m v r} / C_{L}$. For $C_{L m v r} / C_{L}=1$ tip extensions outperform winglets for any weight increase. For a $5 \%$ weight increase the tip extension offers about a $1 \%$ reduction in drag relative to the optimized $90^{\circ}$ winglet and about $0.5 \%$ relative to the $75^{\circ}$ winglet.

However, for the case $C_{L m v r} / C_{L}=2.5$ winglets outperform tip extensions for all weights. A $90^{\circ}$ winglet is optimal for weight increase less than $10 \%$ and a $75^{\circ}$ winglet is optimal for larger weight increases. For a $5 \%$ weight increase the $90^{\circ}$ winglet offers a $1 \%$ reduction in drag as compared to the tip extension.

Our computations are similar to the experimental results reported in Whitcomb's paper ${ }^{4}$ if the same criteria is used. For example, our methods predicts that the Whitcomb winglet offers about a $7 \%$ increase in $\mathrm{L} / \mathrm{D}$ as compared to the reported $9 \%$, and the Whitcomb tip extension offers a $4 \%$ increase in $\mathrm{L} / \mathrm{D}$ as compared to the reported $4 \%$ (These values are obtained at the same reported increase in root bending moment of $3.5 \%$ ).

However, the results are quite different when using integrated bending moment over thickness rather than root bending moment for weight, and when including stall constraints. The chords at the tip of the Whitcomb winglet are smaller than those on the Whitcomb tip extension, leading to a small penalty at $C_{L_{\max }}$. Figure 4 shows the same Pareto front, but include the calculations based on Whitcomb's actual tip extension/winglet geometry with optimized twist across the wing and tip. The Whitcomb winglet is still a better design than the Whitcomb tip extension; however, as seen from the figure, its perhaps not a fair comparison.

Although the analysis shows that for minimum drag the choice of tip extensions versus winglet depends on the ratio $C_{L m v r} / C_{L}$, the difference in drag between the two optimal configurations is not very significant. The choice of whether to use a tip extension or a winglet would more likely be determined by other considerations such as stability and control, structural dynamics, or even marketing.

\section{B. Tip Extensions and Winglets - New Wing Design}

While the previous study held the planform fixed, as appropriate for a retrofit, this study includes the planform in the optimization. The goal is to see if the conclusions for retrofitted wings are similar to those for new wing designs. Rather than using a trapezoidal wing and a trapezoidal tip, we employ a more general description of the geometry by using a larger number of trapezoidal sections. Five trapezoidal sections are 


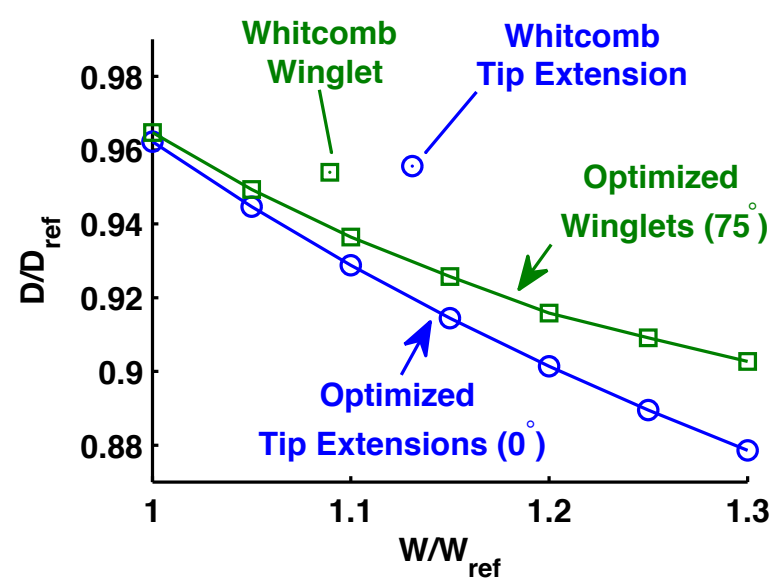

Figure 4. Whitcomb winglet shown to be much closer to an optimal design than Whitcomb tip extension $\left(C_{L_{m v r}} / C_{L}=1.0\right)$.

equally spaced across the wing semi-span, with the tip as one separate trapezoidal section. The following design variables were used: wing span, tip length, chord length at the ends of each section, twist at the ends of each section, and dihedral angle of the tip (Figure 5). As done before, the wing span and the length of the tip are varied parametrically. Transonic drag rise and torsional weight are not included in the computation, so the sweep of the wing is fixed at the value of the reference wing. Since the sensitivity of drag to the tip dihedral angle is often very small, ${ }^{8,14}$ care must be taken to scale this variable properly in order to get correct solutions.

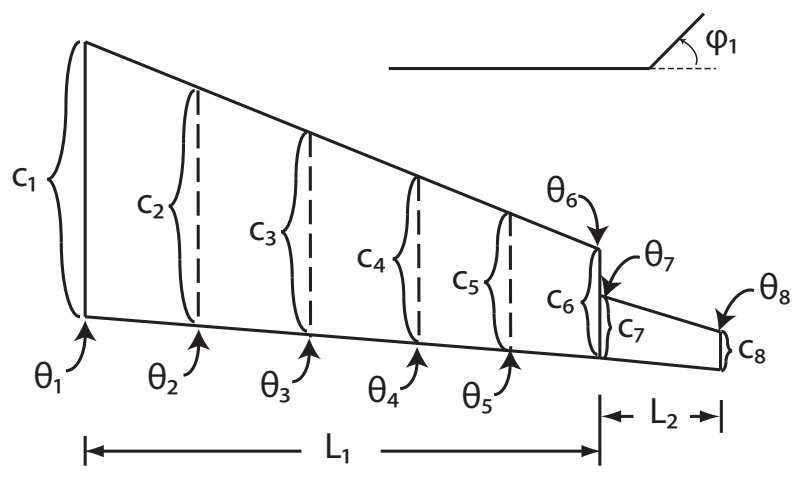

Figure 5. Design variables used in the planform optimization.

The constraints include minimum lift, maximum weight, and minimum stall speed. In addition the lift distribution at the maneuver load is constrained to be positive at every section. A negative maneuver loading can produce negative bending moments at some sections which may lead to negative weight calculations if not handled properly. For this problem negative loading is almost never optimal, but if not constrained can be exploited by the optimizer. This will be explored in more detail later for cases where negative loading may be optimal.

The optimization problem is given as:

$$
\begin{aligned}
\text { min. } & J=\frac{D}{D_{\text {ref }}} \\
\text { w.r.t. } & L_{1}, L_{2},\{c\},\{\theta\}, \phi_{t i p} \\
\text { s.t. } & 1-\frac{L}{L_{\text {ref }}} \leq 0
\end{aligned}
$$




$$
\begin{aligned}
& \frac{W}{W_{\text {ref }}}-1 \leq 0 \\
& \left.\left\{c_{l}\right\}\right|_{C_{\text {Lmax }}}-\left\{c_{\text {lmax }}\right\} \leq 0 \\
& \frac{\left\{\gamma_{\text {mvr }}\right\}}{\bar{\gamma}_{\text {ref }}} \geq 0 \\
& 0.5<\frac{L_{1}}{b_{\text {ref }}}<2 \\
& 0.01<\frac{L_{2}}{b_{\text {ref }}}<0.5 \\
& 0.01<\frac{c}{c_{\text {ref }}}<5 \\
& -20^{\circ}<\frac{\theta}{0^{\circ}}<20^{\circ} \\
& \frac{0^{\circ}}{1000}<\frac{\phi_{t i p}}{1000}<\frac{120^{\circ}}{1000}
\end{aligned}
$$

The reference wing used in this computation (and in the next few sections) is a more modern wing shape than used in the Whitcomb study. The definitions for aspect ratio, taper ratio, and root chord used in Figure 6 are based upon the trapezoidal reference area only. The span of the reference wing is $200 \mathrm{ft}$. Reference conditions are computed at a lift coefficient of 0.4 .

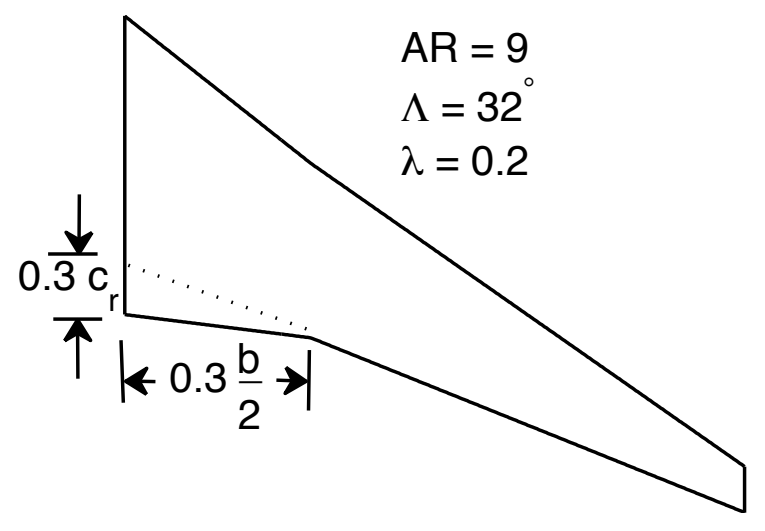

Figure 6. Reference wing geometry used in this and subsequent cases.

The optimized planform shape is shown for a $C_{L m v r} / C_{L}=1$ and 2.5 in Figures $7($ a) and 7 (b) respectively. The optimal tip designs depend on $C_{L m v r} / C_{L}$ in a similar manner to the tip geometries from the last section. For a $C_{L m v r} / C_{L}=1$ the optimal design uses a tip extension and achieves a drag reduction of about $6 \%$ relative to the baseline wing. The span has increased by $6 \%$ of the reference wing span. At $C_{L m v r} / C_{L}=2.5$ the optimal design uses a $85^{\circ}$ dihedral winglet and achieves a drag reduction of about $4 \%$. The wing span is $94 \%$ of the reference wing span, and the winglet length is $20 \%$ of the wing semi-span. However, in this case as well, the difference in lift to drag ratio between optimized winglet designs and tip extension designs is not large. 


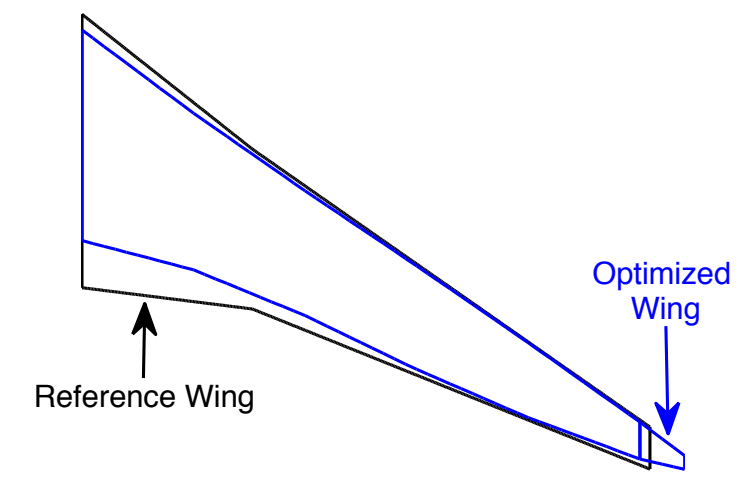

(a) $C_{L m v r} / C_{L}=1.0$, tip extension optimal, $D / D_{\text {ref }}=0.94$

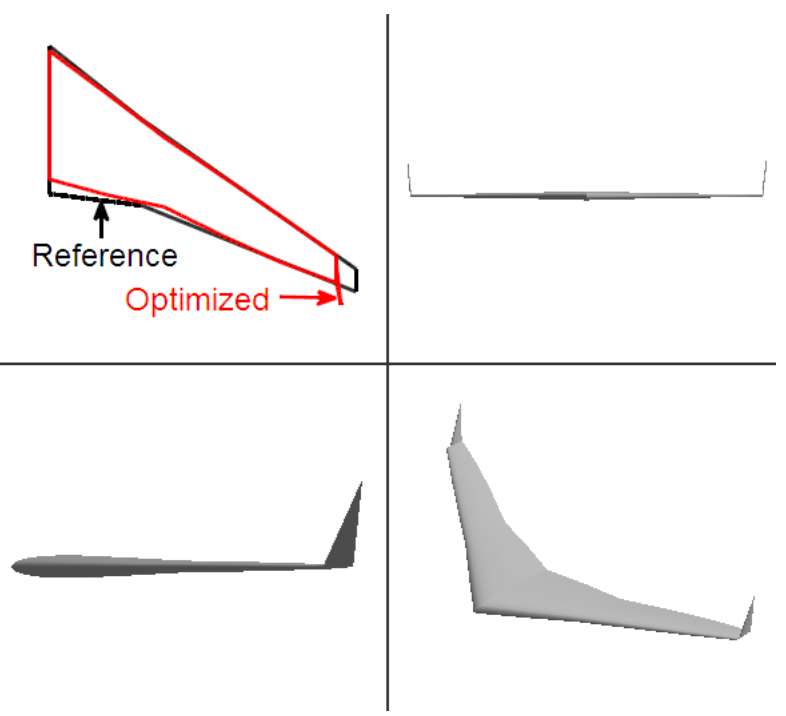

(b) $C_{L m v r} / C_{L}=2.5$, winglet optimal, $D / D_{r e f}=0.96$

Figure 7. Optimized wing and tip geometries.

\section{C-Wings for Tailless Aircraft}

A logical extension to adding a winglet at the end of a wing would be to add another lifting surface at the end of the winglet. Past studies have indeed suggested that C-wings (so named because the shape of the tip resembles a 'C') may be advantageous for certain designs. Von Karman and Burgers showed that for a fixed height and span, the box wing has minimum vortex drag. ${ }^{18} \mathrm{~A} \mathrm{C}$-wing of the same span and height will have very nearly the same inviscid span efficiency, ${ }^{1}$ but when viscous drag is considered the C-wing will certainly have less total drag than the box wing. A study on very large subsonic transports by McMasters et al. discusses other potential benefits for the C-wing configuration besides the high span efficiency. ${ }^{19}$ The nonplanar $\mathrm{C}$-wing is well suited to very large transports due to span constraints. Also, by sweeping the wing or the winglets, the $\mathrm{C}$-wing element can be used for longitudinal control and stability much like a horizontal tail. While these potential benefits are understood, it is not clear what the tradeoffs in performance are once constraints on stability, weight, and stall are imposed. C-wings were examined in the previous cases presented in this paper, but no significant performance advantage was found. For a tailless aircraft however, a C-wing could be beneficial for its effect on the pitching moment about the aerodynamic center.

The pitching moment about an aircraft's center of gravity can be expressed as

$$
C_{m c g}=C_{m a c}-s \cdot m \cdot C_{L}
$$

which should equal zero to trim at the desired lift coefficient. A statically stable aircraft has a positive static margin (aerodynamic center aft of the center of gravity). This means that a stable and trimmed aircraft requires a positive pitching moment about its aerodynamic center. For tailless aircraft, this pitching moment must be provided by the wing. This is often difficult to achieve at reasonable static margins, because conventional airfoil sections have negative section pitching moments. This is one area where a C-wing may provide an advantage compared to a planar flying wing. For an optimally span-loaded C-wing, its C-wing element lifts down. As mentioned, this allows the $\mathrm{C}$-wing element on a swept $\mathrm{C}$-wing to act like a horizontal tail. In this study, trim is enforced by constraining the pitching moment about the aerodynamic center of the wing to be positive. This study also examines zero and negative pitching moments to see the effect this constraint has on the design. Negative pitching moments are generally of limited interest, as these trimmed designs would either be statically unstable or require sections with significant negative camber.

\section{Additional Constraints}

A few modifications must be made to the constraints to properly handle $\mathrm{C}$-wings. The first change is that the local circulation must be allowed to be either positive or negative. In previous cases the loading was 
always constrained to be positive across the wing at the maneuver load. Allowing the loading to be negative introduces several modifications to the constraints.

Negative loading means that the bending moment may be negative at some sections of the wing. This must not lead to negative weight, since the volume of material used to resist bending actually depends on the absolute value of the bending moment. Since we are using a gradient based optimization method, using absolute value is problematic as it leads to discontinuous gradients. This can be avoided, but leads to additional constraints. The number of necessary constraints is reduced by using the observation that negative bending moments will typically only occur near the tip of the wing. Thus, the computation of the bending weight is split into a part due to the inner sections of the wing, and a part due to the outer panels. Since the sign of the bending moment on the outer panels is not known a priori, all possible combinations must be considered. These combinations are put in a vector where the actual bending weight contribution from the outer panels will be the maximum entry in the vector.

$$
W_{b}=I_{\text {binner }}+\max \left\{I_{b}\right\}_{\text {outer }}
$$

Including area dependent weight, the weight constraint is now

$$
I_{\text {binner }}+\left\{I_{b}\right\}_{\text {outer }}+k_{W} S \leq W_{\text {ref }}
$$

A skin thickness constraint is necessary because the optimizer would like to unload the winglet, and drive the winglet to very small chords to reduce drag and weight. But to structurally support the C-wing element this would require very large skin thicknesses in the winglet. The structural model assumes that the skin thickness is much smaller than the airfoil thickness. The skin thickness of the structural box must be constrained to prevent unreasonable designs. The skin thickness is restricted to less than $10 \%$ of the local section thickness.

$$
\left\{t_{s}\right\} \leq 0.1\{t\}
$$

Both skin thickness and section lift coefficients depend on the absolute value of the bending moment. These constraints are broken up into two parts to avoid the use of absolute value.

The final modification used for optimizing $\mathrm{C}$-wing designs is related to the maneuver lift coefficient. Instead of specifying one critical maneuver lift coefficient at a time, as done in the previous cases, the weight is computed at two different maneuver lift coefficients and the weight is taken to be the maximum of the two. More details on the implementation of the constraints are available in an earlier version of this paper. ${ }^{14}$

\section{Weight-Constrained Designs}

For the optimization problem the wing is divided into five trapezoidal sections, one vertical winglet, and one horizontal C-wing element. The design variables include the wing span, the chord lengths at the ends of each section, and the twist at the ends of each section. Since the length of each lifting surface must be varied parametrically, as explained previously, the number of design combinations can become very large. Rather than exhaustively explore this design space, we specify a fixed winglet height and fixed C-wing element length, both $16 \%$ of the wing semi-span. This is a typical height for many modern winglets. The sweep of the main wing is fixed at $32^{\circ}$ and the sweep of the winglet and $\mathrm{C}$-wing element are both fixed at $20^{\circ}$. The optimization problem is given as follows:

$$
\begin{aligned}
\text { min. } & J=\frac{D}{D_{\text {ref }}} \\
\text { w.r.t. } & b,\{c\},\{\theta\} \\
\text { s.t. } & 1-\frac{L}{L_{\text {ref }}} \leq 0 \\
& \frac{\left(I_{\text {binner }}+\left\{I_{b}\right\}_{\text {outer }}+k_{W} S\right)_{C_{L 1}}}{W_{\text {ref }}}-1 \leq 0 \\
& \frac{\left(I_{\text {binner }}+\left\{I_{b}\right\}_{\text {outer }}+k_{W} S\right)_{C_{L 2}}}{W_{\text {ref }}}-1 \leq 0 \\
& \left.\left\{c_{l}\right\}\right|_{C_{L_{\max }}}-\left\{c_{\text {lmax }}\right\} \leq 0
\end{aligned}
$$




$$
\begin{aligned}
& -\left.\left\{c_{l}\right\}\right|_{C_{L_{\text {max }}}}-\left\{c_{l_{\max }}\right\} \leq 0 \\
& \left.\left\{t_{s}\right\}\right|_{C_{L 1}}-0.1\{t\} \leq 0 \\
& \left.\left\{t_{s}\right\}\right|_{C_{L 2}}-0.1\{t\} \leq 0 \\
& -\left.\left\{t_{s}\right\}_{\text {outer }}\right|_{C_{L 1}}-0.1\{t\} \leq 0 \\
& -\left.\left\{t_{s}\right\}_{\text {outer }}\right|_{C_{L 2}}-0.1\{t\} \leq 0 \\
& C_{\text {mac }}-C_{\text {mac target }}=0 \\
& 0.5<\frac{b}{b_{\text {ref }}}<5 \\
& 0.01<\frac{c}{c_{\text {ref }}}<5 \\
& -20^{\circ}<\theta<20^{\circ}
\end{aligned}
$$

Three different constraints on pitching moment about the aircraft aerodynamic center are explored: $C_{\text {mac target }}=+0.1,0,-0.1$. As mentioned, typically a positive $C_{m a c}$ is desirable. A $C_{m_{a c}}$ of +0.1 , for example, would allow a flying wing with symmetric sections and a total lift coefficient of 1 to trim with a static margin of $10 \%$, or a cambered section flying wing with section pitching moments of -0.1 to trim with zero static margin. This analysis assumes symmetric sections for simplicity.

For each target $C_{m a c}$, the optimal C-wing is compared to an optimized wing with winglet and an optimized wing subject to the same constraints. The minimum drag solution for each configuration is shown in Table 2 normalized by the drag of the reference wing. Table 3 lists the optimized span of each configuration normalized by the span of the reference wing. The reference wing in these cases is the same as in the previous section (Figure 6).

Table 2. Relative drag $\left(D / D_{r e f}\right)$ of optimized configurations - weight constrained, trimmed

\begin{tabular}{|c|ccc|}
\hline$C_{\text {mac }}$ & Wing & Wing + Winglet & C-wing \\
\hline+0.1 & 1.091 & 1.095 & $\mathbf{1 . 0 8 1}$ \\
0 & 0.978 & $\mathbf{0 . 9 6 4}$ & 0.971 \\
-0.1 & 1.345 & $\mathbf{1 . 3 1 6}$ & 1.321 \\
\hline
\end{tabular}

Table 3. Relative spans $\left(b / b_{r e f}\right)$ of optimized configurations - weight constrained, trimmed

\begin{tabular}{|c|ccc|}
\hline$C_{\text {mac }}$ & Wing & Wing + Winglet & C-wing \\
\hline+0.1 & 1.078 & 1.076 & 0.977 \\
0 & 1.00 & 0.956 & 0.947 \\
-0.1 & 0.959 & 0.907 & 0.902 \\
\hline
\end{tabular}

Table 2 shows that trimming a tailless aircraft has a profound effect on the drag. This can be seen quite easily from a simple analysis relating the lift centroid to the minimum induced drag of the wing. An elliptically loaded wing has a lift centroid at $42.4 \%$ semi-span. For a swept back wing, shifting the lift centroid inboard would provide a greater pitching moment about the aerodynamic center. The new location of the lift centroid relative to the elliptic wing would be

$$
\begin{aligned}
\eta_{c} & =0.424-\frac{\Delta y}{b / 2} \\
& =0.424-\frac{2 \Delta x}{b \tan \Lambda}
\end{aligned}
$$

Then relating the change in streamwise shift in lift centroid to the change in pitching moment about the aerodynamic center $\left(\Delta C_{m a c}=\frac{\Delta x}{c} C_{L}\right)$

$$
\eta_{c}=0.424-\frac{\Delta C_{m a c}}{C_{L}} \frac{2 c}{b \tan \Lambda}
$$




$$
=0.424-\frac{\Delta C_{m a c}}{C_{L}} \frac{2}{A R \tan \Lambda}
$$

If we let $\Delta C_{m_{a c}}=0.1, C_{L}=0.4, A R=9$, and $\Lambda=32^{\circ}$ the lift centroid is now $\eta_{c}=0.335$.

The minimum induced drag for a given lift and location of the lift centroid is given by ${ }^{12}$

$$
e_{\text {inviscid }}=\left[\frac{9}{2} \pi^{2} \eta_{c}^{2}-12 \pi \eta_{c}+9\right]^{-1}
$$

which for the present example gives $e_{\text {inviscid }}=0.74$. This corresponds to a $35 \%$ increase in induced drag or roughly a $17 \%$ increase in total drag, just due to trimming the aircraft.

This rough estimate is consistent with the large drag difference between trimming at $C_{m a c}=0$, and trimming at $C_{m a c}=0.1$. For the later case, however the $\mathrm{C}$-wing provides a small drag advantage. It has about $1 \%$ less drag, and a $9 \%$ smaller span relative to the optimized wing. The geometry for the optimized $\mathrm{C}$-wing is shown in Figure 8(a). The winglet and C-wing element however, have small chords which require additional assessment of the structural dynamics. The lift distributions for this design is shown in Figure 8(b). The coordinate $\xi$ traverses the wing tangentially, and the sign convention for positive section $c_{l}$ is defined by having positive circulation point in the same direction everywhere on the wing. As expected, the C-wing element is lifting downward (positive section $c_{l}$ ) The pitching moment constraint is met by shifting the load far inboard, although the $\mathrm{C}$-wing element does provide some nose-up pitching moment.

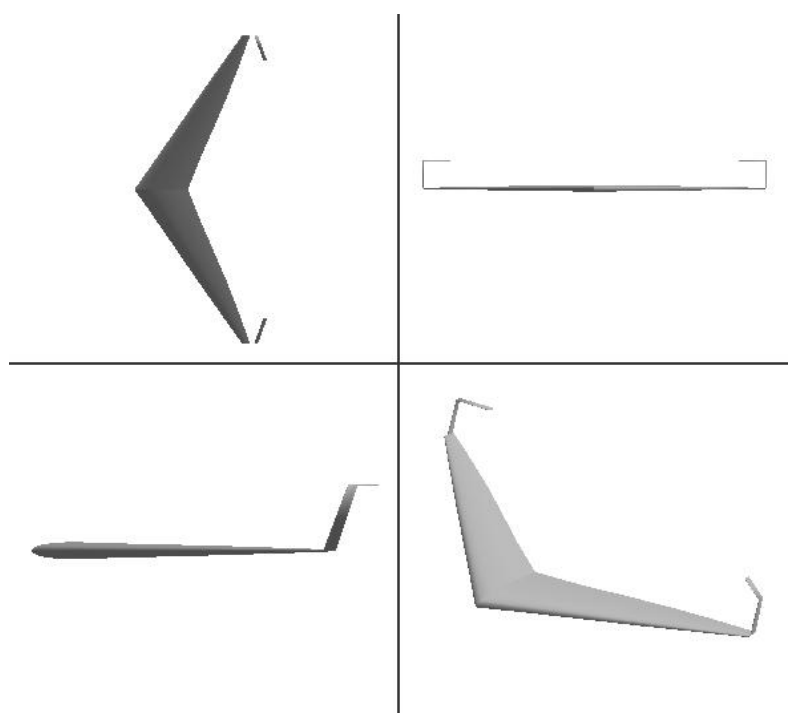

(a) Wing geometry

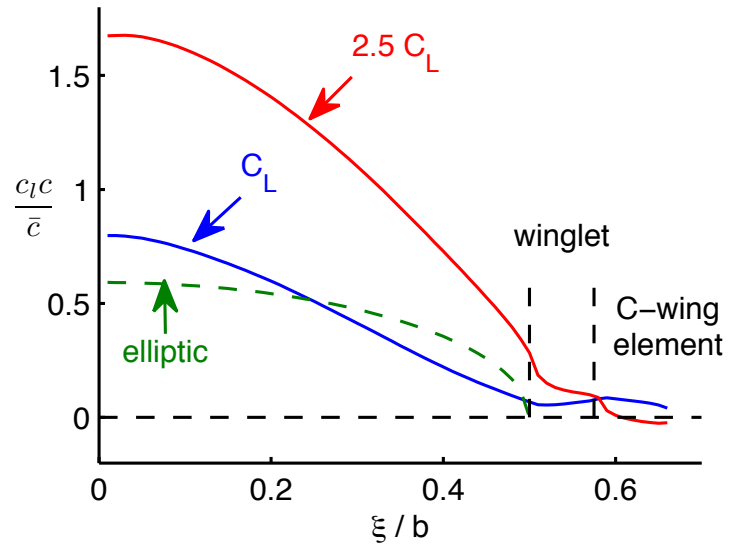

(b) Lift distribution at cruise lift coefficient and at 2.5 times cruise lift coefficient.

Figure 8. Optimized wing for tailless aircraft (C-wing) with positive pitching moment about the aerodynamic center constraint $\left(C_{m_{a c}}=+0.1\right)$.

For the constraint $C_{m a c}=0$ the optimal design is a wing with a winglet. The optimal wing with winglet reduces the drag by about $1.5 \%$ and has a $5 \%$ smaller span relative to the optimized wing. The optimal wing with winglet is seen in Figure 9(a). The lift distribution for this configurations is seen in Figure 9(b). The lift distribution is nearly elliptic.

For $C_{m a c}=-0.1$ the optimal design is also a wing with a winglet. The optimal geometry is seen in Figures 10(a). The wing with winglet design achieves a drag reduction of $2 \%$, and has a $5 \%$ smaller span relative to the optimized wing. The lift distribution for the configuration is seen in Figure 10(b). The lift distribution is heavily shifted outboard to satisfy the pitching moment constraint. The wing has rather large chords inboard to carry the bending weight. The large changes in chord makes some of our assumptions suspect. Three-dimensional viscous drag effects make the section viscous drag buildup method less accurate. Also the large chord variation makes the assumption of a constant $t / c$ a poor approximation. Further analyses would be required for these designs to more completely assess their relative benefits.

In all three cases, however, the difference in drag between the wing, wing with winglet, and C-wing never 


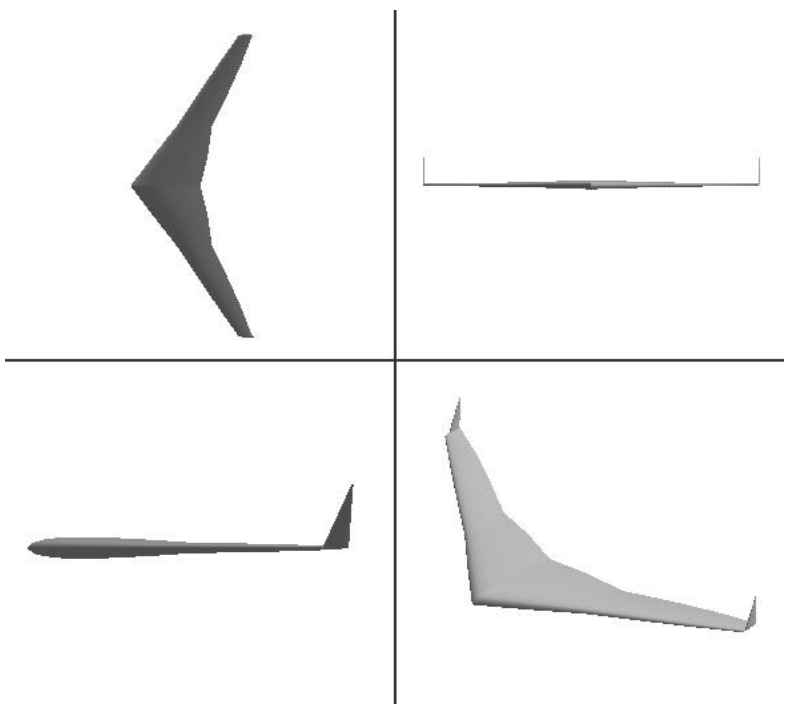

(a) Wing geometry

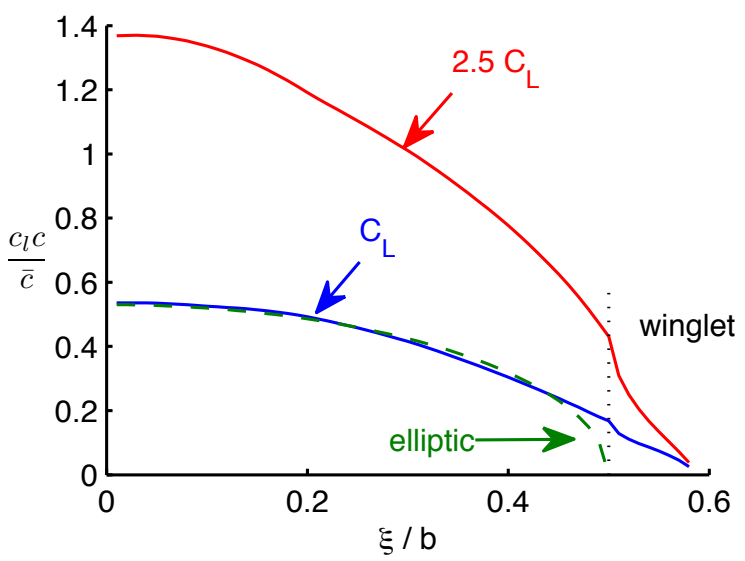

(b) Lift distribution at cruise lift coefficient and at 2.5 times cruise lift coefficient.

Figure 9. Optimized wing for tailless aircraft (wing with winglet) with zero pitching moment about the aerodynamic center constraint $\left(C_{m_{a c}}=0\right)$.

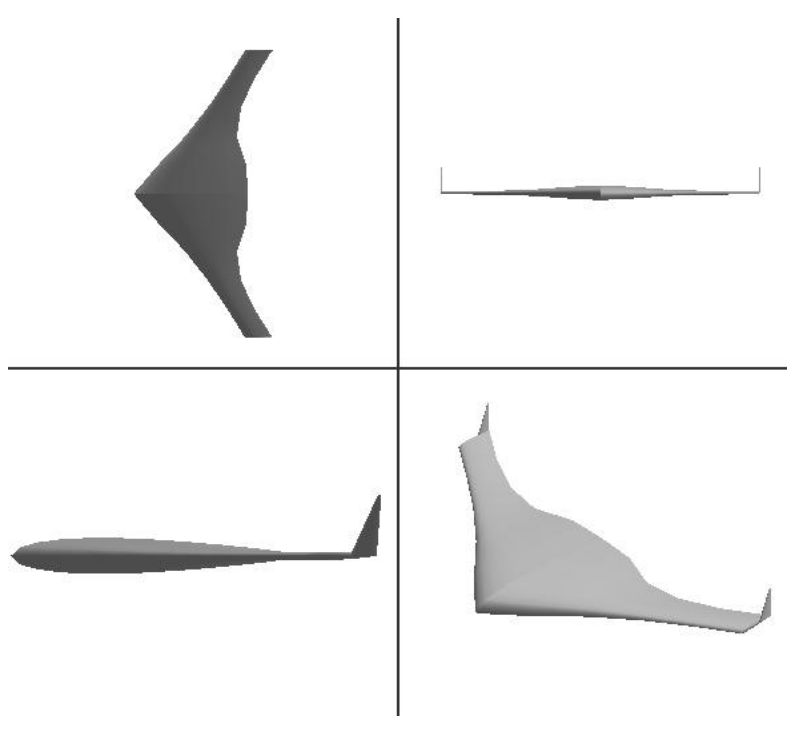

(a) Wing geometry

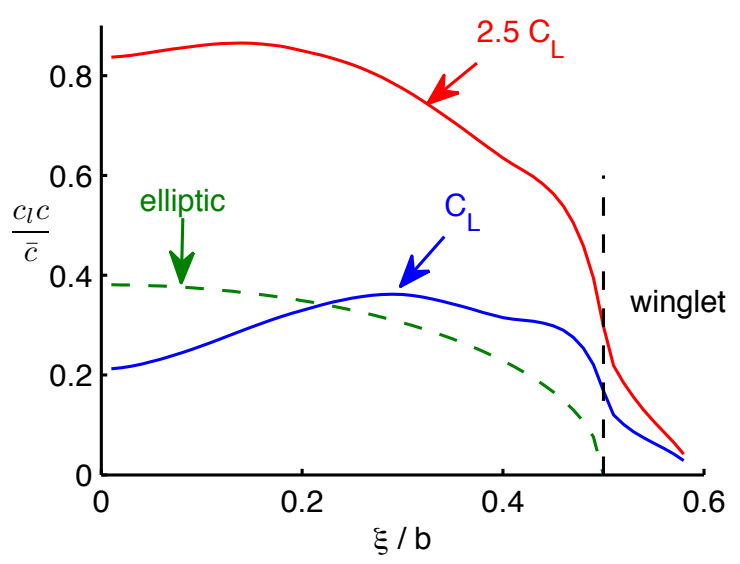

(b) Lift distribution at cruise lift coefficient and at 2.5 times cruise lift coefficient.

Figure 10. Optimized wing for tailless aircraft (wing with winglet) with negative pitching moment about the aerodynamic center constraint $\left(C_{\text {mac }}=-0.1\right)$. 
differs by more than a percent or two. The configuration choice would likely be dictated by something other than drag such as span, structural dynamics, or radar cross section.

\section{Span-Constrained Designs}

The previous figures show that the variation in drag is small between the different configurations at a given pitching moment constraint, however the variation in span is much larger. Constraining the span, rather than the weight, may lead to different conclusions for optimal designs. Winglets can be used to improve performance for designs with span constraints, ${ }^{1}$ but it is not clear if C-wings add any additional benefit for span-constrained wings. We compare optimized wings, wings with winglets, and $\mathrm{C}$-wings, all constrained to have a span less than or equal to that of the reference wing. Rather than fix the weight at a particular value, the results are presented as a Pareto front. As mentioned before, the winglet height and $\mathrm{C}$-wing element length are fixed at $16 \%$ of the wing semi-span.

Without span constraints, a C-wing with a pitching moment constraint of $C_{m a c}=+0.1$ provides a small performance benefit relative to wings with winglets. For span-constrained designs, the analysis shows that the performance advantage of the C-wing is a bit larger (Figure 11). At a fixed span and weight, the optimized C-wings have from $1 \%$ to $3 \%$ less drag than the optimized wings with winglets at weights ranging from $0 \%$ to $50 \%$ greater than that of the reference wing. For the cases of $C_{m_{a c}}$ equal to 0 and -0.1 (not shown here), there is still no aerodynamic benefit to C-wings as compared to wings with winglets.

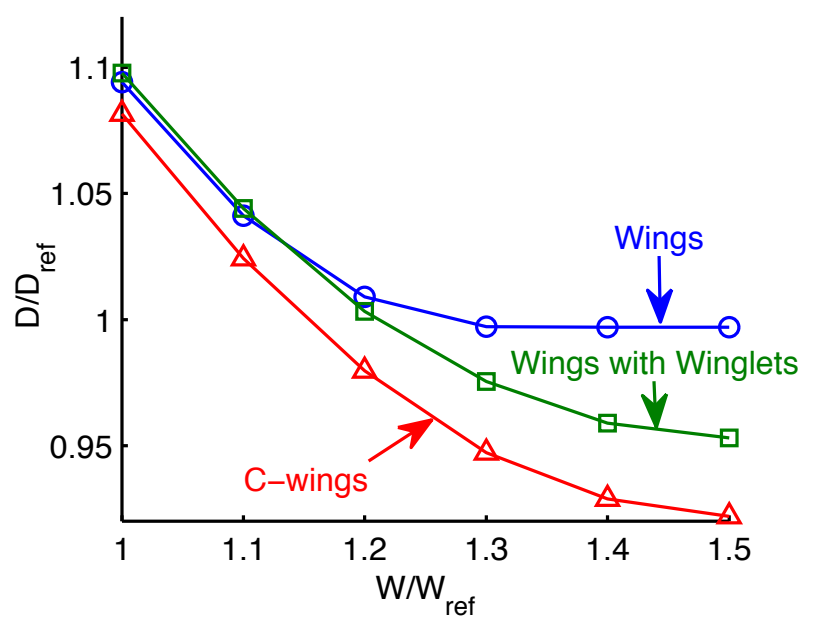

Figure 11. Pareto front for span-constrained tailless aircraft. For a fixed span and weight C-wings show more significant performance benefits once trim and stability constraints are imposed $\left(C_{m_{a c}}=0.1\right)$.

\section{Effect of Sweep on C-wing Designs}

The results discussed so far have been for a relatively highly swept wing ( $32^{\circ}$ quarter chord sweep) to account for transonic effects. However, low sweep wings may be used even in transonic conditions through careful shape optimization. This may be preferable for some configurations in order to promote natural laminar flow or to increase the structural efficiency.

At low sweep angles, flying wing designs must employ even greater washout to provide nose up pitching moment while $\mathrm{C}$-wing designs can increase the loading on the $\mathrm{C}$-wing element to provide nose up pitching moments without the need for as much washout. Thus, we expect the benefits of $\mathrm{C}$-wings may be greater for low sweep designs. Preliminary analysis suggests that this is the case, but the effects of sweep and large changes in chord are not captured in sufficient fidelity in the present model to quantify the results accurately. With a fixed quarter chord sweep, and the winglet aligned with the trailing edge of the wing tip, the optimizer would like to increase the tip chord so as to move the winglet and C-wing element further aft. This allows a greater moment arm for the pitching moment. The decrease in necessary washout more than compensates for the decrease in span due to larger tip chords. 


\section{Maneuver Load Alleviation}

The same set of tools described here can be used to analyze a much broader range of conceptual wing design problems than the tip design cases presented so far. Many wing design problems have the same important objectives and constraints relating to drag, lift, weight, stability and trim, and stall speed. As one example, the methodology is applied to planar wings designed with active load alleviation.

Many of the previous results have shown how the conditions at the maneuver load affect the optimal design. In particular, for a $C_{L m v r} / C_{L}$ other than 1 , it is clear that the additional lift distribution is tailored to balance an efficient cruise load with an inboard loaded maneuver load. If we are also able to change the basic lift distribution (i.e. dynamically twist the wing), then the difference between an ideal cruise load distribution and an ideal structural limit load distribution can be exploited even further. Here, it is assumed that the aircraft can provide gust load alleviation in a similar manner, but that the structure is critical at maneuver.

For simplicity, this analysis is for a planar wing with five trapezoidal sections and no tip devices. The design variables include span, chord distribution, and twist distribution. In addition, the twist distribution at the maneuver load must be included. The cruise load distribution and structural limit load distribution are then only coupled through the planform geometry, and not through the twist distribution. The objective is to minimize drag at cruise subject to constraints on cruise lift, maneuver lift, maneuver weight, and stall speed. Additionally, the maneuver lift distribution must not stall. Since the wing is planar, the weight calculation can be simplified by fixing the wetted area so that only load dependent weight need be considered. The optimization problem is given as:

$$
\begin{aligned}
& \text { min. } \quad J=\frac{D}{D_{\text {ref }}} \\
& \text { w.r.t. } \quad b,\{c\},\{\theta\},\{\theta\}_{m v r} \\
& \text { s.t. } \quad 1-\frac{L}{L_{r e f}} \leq 0 \\
& 1-\frac{L_{m v r}}{2.5 L_{r e f}} \leq 0 \\
& \frac{W_{\text {mvr }}}{W_{\text {ref }}}-1 \leq 0 \\
& \left.\left\{c_{l}\right\}\right|_{C_{L_{\text {max }}}}-\left\{c_{l_{\text {max }}}\right\} \leq 0 \\
& \left.\left\{c_{l}\right\}\right|_{C_{L_{\text {mvr }}}}-\left\{c_{l_{\text {max }}}\right\} \leq 0 \\
& \frac{\left\{\gamma_{m v r}\right\}}{\bar{\gamma}_{\text {ref }}} \geq 0 \\
& \frac{S}{S_{\text {ref }}}-1=0 \\
& 0.5<\frac{b}{b_{\text {ref }}}<5 \\
& 0.01<\frac{c}{c_{r e f}}<5 \\
& -20^{\circ}<\theta<20^{\circ} \\
& -20^{\circ}<\theta_{m v r}<20^{\circ}
\end{aligned}
$$

Since the twist distribution at maneuver is independent of the twist distribution at cruise, the parameter $C_{L m v r} / C_{L}$ is no longer important. Instead the parameter $c_{l m a x} / C_{L m v r}$ governs the design. This parameter is like $c_{l \max } / C_{L \max }$, since stall becomes critical at maneuver as well as at $C_{L \max }$, and reflects the degree to which the maneuver lift distribution is constrained by stall. This ratio approaches one (flatter $c_{l}$ distribution) as the critical structural altitude is increased, or as the cruise lift coefficient is increased at fixed altitudes.

The reduction in drag from using maneuver load alleviation (MLA) is shown in Figure 12. As $c_{l \max } / C_{L \operatorname{mvr}}$ is increased even greater drag reductions are possible. However, designs with active load alleviation tend toward very large aspect ratios. In those cases, flutter becomes an important consideration not accounted for here. Thus, in this paper, we limit our attention to cases where $c_{\operatorname{lmax}} / C_{L \text { mvr }}$ is close to one. This may 
be excessive for realistic designs, but illustrates the trend. For these cases, using maneuver load alleviation offers about $15-20 \%$ drag savings.

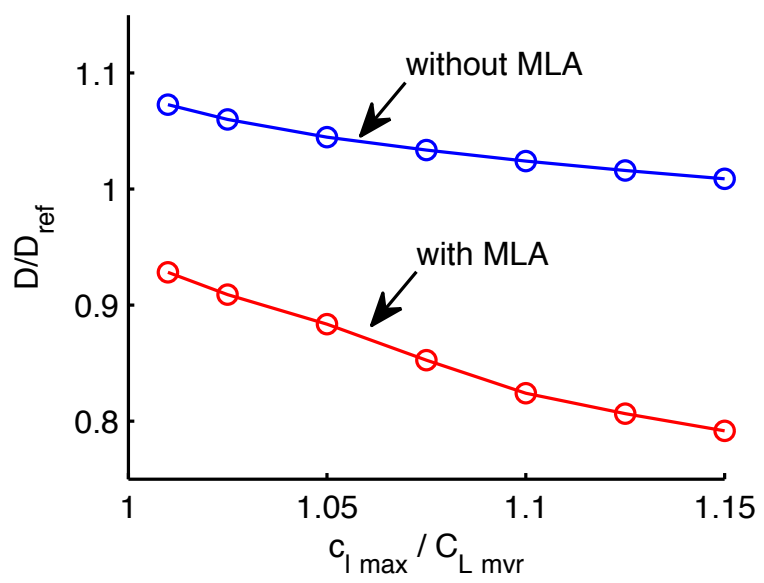

Figure 12. Drag reduction as a function of $c_{l \max } / C_{L m v r}$ (wings constrained not to stall at cruise or maneuver load). MLA shows drag savings on the order of $15-20 \%$.

As an example, the case $c_{l \max } / C_{L m v r}=1.05$ is presented. The optimal planform with and without maneuver load alleviation is shown in Figure 13(a). Stall constraints really limit the wing designed without maneuver load alleviation at this condition, forcing a span smaller than the reference wing. However, the wing designed with maneuver load alleviation is able to increase the span out to an aspect ratio of 13.5 (based on the trapezoidal area of the reference wing). The large increase in span is achieved by shifting the lift inboard with maneuver load alleviation as seen in Figure 13(b).

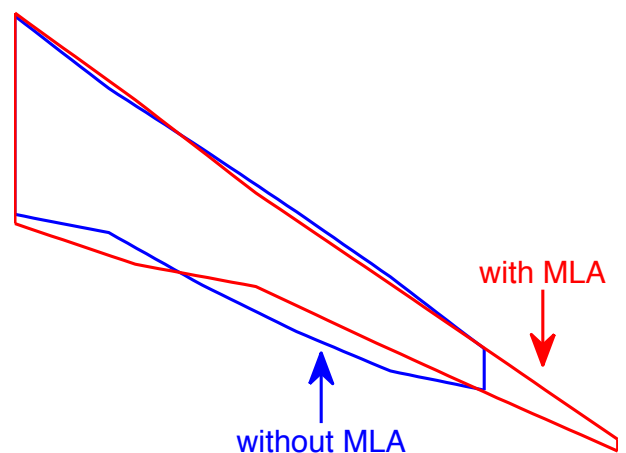

(a) Planform shape

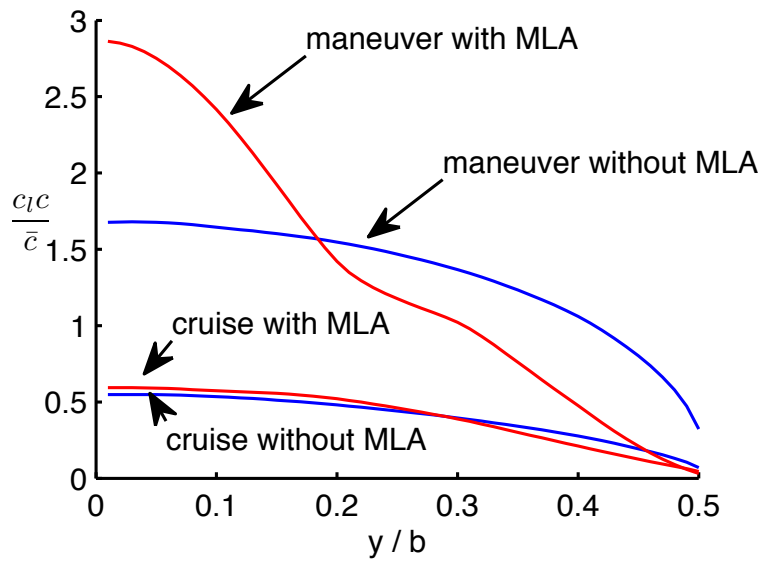

(b) Cruise and maneuver load distributions

Figure 13. Optimized planar wing with and without MLA $\left(c_{l \max } / C_{L \text { mvr }}=1.05\right)$.

\section{Conclusion}

The results demonstrate several important considerations in conceptual wing design including the importance of accounting for the wing structural box, viscous drag, section $c_{l}$ constraints, differences in cruise loading and maneuver loading, wing skin thickness, and the benefit of using multidisciplinary optimization in the conceptual design phase. Some of the most significant results presented here include:

- At fixed lift, weight, and stall speed, the minimum drag trapezoidal tip device depends on the ratio of the maneuver lift coefficient to the cruise lift coefficient. For $C_{L m v r} / C_{L}=1$ tip extensions show a 
slight advantage compared with winglets, while for $C_{L m v r} / C_{L}=2.5$ winglets are slightly better than tip extensions. These trends apply both for wing retrofits and for new wing designs.

- C-wings for tailless aircraft have slightly lower drag compared with wings and wings with winglets when stability and trim constraints are imposed. This may be especially true for designs with span constraints or low sweep.

- Maneuver Load Alleviation (and Gust Load Alleviation) can offer significant reductions in drag (on the order of $15 \%$ for the designs evaluated here); however, an analysis of the structural dynamics is necessary for the resulting high aspect ratio designs.

These conclusions represent fundamental multidisciplinary considerations in wing design. Design decisions, however, would need to be made in the context of specific and complete design studies. For example, as pointed out by Heyson et al., it is not clear that an increase in wing weight will lead to an increase in total aircraft weight. It may be that the increase in wing weight is offset by the decrease in fuel weight due to the reduction in drag. Many more complex trade-offs exist as entire configurations and missions are optimized. Although this paper is meant to explore results of a more general nature, the methodology can be applied in specific conceptual design studies as the methods allows for efficient exploration of large areas of the design space.

A few extensions to the method are currently being pursued. Transonic drag rise, airfoil section design, variation in material properties (especially in winglets), torsion-dependent weight (may be particularly important for raked tips), and steady aeroelastics are a few areas that may yield additional insights.

\section{Acknowledgments}

The authors gratefully acknowledge support from the National Defense Science and Engineering Graduate (NDSEG) Fellowship program.

\section{References}

\footnotetext{
${ }^{1}$ Kroo, I., "Nonplanar Wing Concepts for Increased Aircraft Efficiency," VKI Lecture Series on Innovative Configurations and Advanced Concepts for Future Civil Aircraft, June 2005.

${ }^{2}$ Hemke, P. E., "Drag of Wings with End Plates," TR-267, NACA, 1928.

${ }^{3}$ Mangler, W., "The Lift Distribution of Wings with End Plates," TM-856, NACA, 1938.

${ }^{4}$ Whitcomb, R. T., "A Design Approach and Selected Wind-Tunnel Results At High Subsonic Mounted Speeds for Winglets," TN-D-8260, NASA, July 1976.

${ }^{5}$ Heyson, H. H., Riebe, G. D., and Fulton, C. L., "Theoretical Parametric Study of the Relative Advantages of Winglets and Wing-Tip Extensions," TP-1020, NASA, September 1977.

${ }^{6}$ Flechner, S. G. and Jacobs, P. F., "Experimental Results of Winglets on First, Second, and Third Generation Jet Transports," CTOL Transport Technology, Langley Research Center, Jan. 1978, pp. 553-569.

${ }^{7}$ Jones, R. T. and Lasinski, T. A., "Effect of Winglets on the Induced Drag of Ideal Wing Shapes," TM-81230, NASA, September 1980

${ }^{8}$ Kroo, I., "Design and Analysis of Optimally-Loaded Lifting Systems," AIAA 84-2507, October 1984.

${ }^{9}$ Asai, K., "Theoretical Considerations in the Aerodynamic Effectiveness of Winglets," Journal of Aircraft, Vol. 22, No. 7 , July 1985, pp. 635-637, doi:10.2514/3.45177.

${ }^{10}$ Zimmer, H., The Aerodynamic Optimization of Wings at Subsonic Speeds and the Influence of Wing Design, Ph.D. thesis, University of Stuttgart, Stuttgart, Germany, 1983, Translated into English in NASA TM-88534.

${ }^{11}$ Slingerland, R. and Verstraeten, J. G., "Drag Characteristics for Optimally Span-loaded Planar, Wingletted, and Cwings," 46th AIAA Aerospace Sciences Meeting and Exhibit, AIAA, January 2008.

${ }^{12}$ Jones, R. T., "The Spanwise Distribution of Lift for Minimum Induced Drag of Wings Having a Given Lift and a Given Bending Moment," TN-2249, NACA, December 1950.

${ }^{13}$ McGeer, T., Wing Design for Minimum Drag with Practical Constraints, Ph.D. thesis, Stanford University, Dec. 1983.

${ }^{14}$ Ning, S. A. and Kroo, I., "Tip Extensions, Winglets, and C-wings: Conceptual Design and Optimization," 26th AIAA Applied Aerodynamics Conference, AIAA, August 2008.

${ }^{15}$ Weissinger, J., "The Lift Distribution of Swept-Back Wings," TM-1120, NACA, March 1947, Translation of Uber die Auftriebsverteilung von Pfeilflugeln Forschungsbericht Nr. 1553.

${ }^{16}$ Federal Aviation Administration, FAR Part 25-337.

${ }^{17}$ Lowson, M. V., "Minimum Induced Drag for Wings with Spanwise Camber," Journal of Aircraft, Vol. 27, No. 7, July 1990, pp. 627-631, doi:10.2514/3.56861.

18 von Karman, T. and Burgers, J. M., Airfoils and Airfoil Systems of Finite Span, Vol. 2 of General Aerodynamic TheoryPerfect Fluids, Springer (Berlin), 1935.
} 
${ }^{19}$ McMasters, J. H., Paisley, D. J., Hubert, R. J., Kroo, I., Bofah, K. K., Sullivan, J. P., and Drela, M., "Advanced Configurations for Very Large Subsonic Transport Airplanes," CR-201614, NASA, October 1996. 